\title{
Medication errors and professional practice of registered nurses
}

\author{
Cecil Deans, University of Ballarat and Ballarat Health Services
}

This Australian study identified and described the incidence of medication errors among registered nurses, the type and causes of these errors and the impact that administration of medications has on the professional practice of registered nurses. Mostly, medication errors were attributed to documentation issues, including: illegible handwriting, misunderstanding abbreviations, misplaced decimal point, misreading and misinterpreting written orders. Several human factors were attributed to potential causes of medication errors, including: stress, fatigue, knowledge and skill deficits. Environmental factors, namely, interruptions and distractions during the administration of medications, were also attributed to potential errors. The study found professional nursing practice involving administration of medications had a strong education, patient and ethical focus. Over a quarter of the respondents indicated that further training in medication administration would positively impact on their nursing practice. The registered nurses also highlighted they would appreciate more time to spend with patients when administering medications. Medication errors are not the sole responsibility of any single professional group, therefore, collaboration with other health professionals is central to establishing processes, policies, strategies and systems that will reduce their occurrence. The organisation and those nurses employed within it share an accountability to ensure safe administration of medications to patients. Based on study results, several recommendations are directed towards preventing or reducing medication errors and supporting nurses in providing best practice.

Key words: medication errors, professional practice, registered nurses
Clinical Nursing, University of Ballarat and Ballarat Health Services Email: c.deans@ballarat.edu.au

\section{Introduction}

Although the process of delivering medications to patients involves a number of steps requiring collaboration between medical practitioners, registered nurses and pharmacists, it is the primary responsibility of nurses to ensure the safe administration of medication. According to Anderson and Webster (2001) administration of medication is probably the highest-risk task a nurse can perform, and accidents can lead to devastating consequences for the patient and for the nurse's career. Mayo and Duncan (2004) suggest that the psychological trauma caused by committing a medication error can be overwhelming to a nurse, '...[they] may feel upset, guilty, and terrified' (p210). Carothers (1998) stated that 'medication errors not only cause harm to patients, they also mar the reputation of all health professionals in whom patients place their trust' (p104).

In the past 10 years, medication errors have become recognised as an important cause of iatrogenic disease in hospital patients (Ross et al 2000). At a national level, the Australian Council for Safety and Quality in Health Care (2001) has identified medication safety as a priority area and is progressing the development of national strategies to reduce adverse medication events. In Victoria, The Royal Melbourne Hospital Inquiry (RMH) identified a number of issues related to the safe administration of medicines by nurses (2002). In response to the Commissioner's concerns, medication management has become a major focus of nursing and Clinical Risk Management (CRM).

\section{Literature review}

In an American study, Phillips et al (2001) reviewed all cases of medication errors reported to the Food and Drug Administration for a six year period (1993-1998) and found that out of 5,366 medication reports, $68.2 \%$ resulted in serious patient outcomes and $9.8 \%$ were fatal. Four hundred and sixty nine deaths revealed 594 errors with $40.9 \%$ being administration of an improper dose, followed by $16 \%$ being administration of the incorrect drug to the patient. Fifty seven errors $(9.5 \%)$ were caused by administering a drug by the incorrect route. The five major categories of causes were (1) communication (2) name confusion (3) labelling (4) human factors, and (5) packaging. The most common causes of medication errors resulting in death were human factors $(65.2 \%)$ followed by communication, transcription and handwriting errors (15.8\%). A study by Leape et al (1995) 
reported that $39 \%$ of errors occurred at the physician ordering stage and $38 \%$ at the nurse administration stage. According to Leape et al, up to $70 \%$ of all physician ordering errors are intercepted by pharmacists and nurses prior to patient administration; regrettably however, there is no similar human safety net for nurses when medications are administered to the patient.

Benner et al (2002) investigated errors made by nurses in 2002. They categorised 21 case studies of nursing errors from the disciplinary files of nine State Boards of Nursing in the USA. Eight categories represented the broad range of possible errors and their contributive or causative factors. One of the eight categories identified was medication errors and constituted seven types of error:

1. Missed doses of medication.

2. Wrong time of administration (60 minutes before or after the prescribed time).

3. IV rate too fast, or too slow.

4. Wrong concentration of dosage of medication delivered via intra-venous route.

5. Wrong route of administration.

6. Wrong medications delivered due to mis-identifying the patient.

7. Wrong medications administered.

Osborne et al (1999) conducted a study in America of nurses' perceptions of medication errors and reported the main cause was failure to identify the patient $(35 \%)$ followed by fatigue and exhaustion $(25 \%)$. Results also revealed the majority of nurses noted that most medication errors were not reported because of fear of reaction from nurse managers and co-workers. Mayo and Ducan (2004) conducted a survey of 983 American nurses and asked them to rank causes of medication errors as perceived by participants. Results showed the top three ranked (out of 10) perceived causes of drug errors were (1) doctors' handwriting is difficult to read or illegible, (2) nurses are distracted, and (3) nurses are tired and exhausted. When participants were asked to estimate what percent of all drug errors are reported to the nurse manager by the completion of an incident report, less than half of the nurses believed that all drug errors are reported.

In Australia, Walker and Lowe (1998) focused on nurses' views of reportable medication incidents and explored nurses' beliefs about medication incident reporting. They found that nurses were more likely to report a medication error when they believed patient safety may have been compromised and less likely to report for errors of documentation and minor variations from the prescription. An interesting finding from this study was that nurses frequently said self-preservation was a major reason for nurses choosing not to report medication errors. This fear of being blamed has been documented by Reason (2000) who classified it under the 'person-centred approach', which views errors arising from 'forgetfulness, inattention, poor motivation, carelessness, negligence, and recklessness'. According to Walker and Lowe (1998) attention to individuals rather than processes can result in nurses having a genuine fear of getting into trouble and receiving a reprimand from those in authority. Alternatively, Brown (2001) advocated for the systems approach, which emphasises the human condition of fallibility and anticipates that errors will occur. Brown suggested the focus for this approach is on identifying predisposing factors within the working environment or system that result in error commission.

Shea (1999) conducted a review of the literature on medication errors and highlighted the mathematical ability of nurses, their workload, nursing care delivery systems and staffing levels on different shifts as factors to be borne in mind by nurse managers - and the need for nurse educators to provide in-service education relating to medication. Another significant conclusion was the multidisciplinary nature of medication errors and the need for a multidisciplinary approach in reducing errors.

\section{Research aim and questions}

The aim of the survey was to identify and describe the incidence, type and causes of medication errors and impact that the administration of medications has on professional practice of registered nurses at a major regional hospital in Victoria. In addressing the research aim, the study answered the following research questions:

1. What is the incidence among nurses of medication errors when administering medications to patients?

2. What types of medication errors are made?

3. What are the causes of medication errors as perceived by registered nurses?

4. How do registered nurses perceive their practice when administering medications?

\section{Method}

The study employed an anonymous self-report survey to collect data from registered nurses employed in selected settings at a major regional hospital in Victoria. Medication errors were defined as 'any preventable event that may cause or lead to inappropriate medication use or patient harm while the medication is in the control of the health care professional, patient, or consumer' (National Coordinating Council for Medication Error Reporting and Prevention 1999: NCCMERP).

\section{Setting and sample}

Six clinical areas were selected as the setting for the study. Three of the clinical areas were designated as surgical units, two as medical and one area was designated as a palliative care facility. A total of 154 registered nurses employed in the study settings were surveyed.

\section{Instrument}

The Medication Error Questionnaire (MEQ) was developed by the researcher and is based upon the National Coordinating Council for Medication Error Reporting and Prevention (NCC 
Medication errors and professional practice of registered nurses MERP) Taxonomy of Medication Errors. The purpose of the NCC MERP taxonomy is to provide a standard language and structure of medication error-related data for use in developing data bases analysing medication error reports. The MEQ is a self-report questionnaire with 12 items requesting nurses to report on medication errors in the past four weeks. The MEQ also has five sections that solicit information about what registered nurses think was the cause of the medication error and one item asking whether they reported the error. The final section of the MEQ has eight items constructed as a Likert-type scale with response sets ranging from Strongly Agree to Strongly Disagree, to questions that identified how drug administration impacted upon registered nurses' level of satisfaction, autonomy and confidence. Content validity of the MEQ was reviewed by six senior nurse managers, six nurse unit managers and a senior pharmacist who agreed the instrument was appropriate to the research questions. Ethics approval was gained from the hospital's Ethics Committee. Respondents were assured of anonymity and confidentiality in that no identifying information would be contained in the study instruments.

\section{Procedure}

The MEQ was distributed by internal mail to all registered nurses rostered on duty in the selected research settings.

\section{Results}

Seventy nine MEQs were completed, representing a $51 \%$ response rate. The MEQ results showed a number of medication errors had occurred over the four week timeframe. Table 1 (below) shows the frequency and percentage of error types. A number of nurses did not complete every section in the MEQ, therefore percentages do not always add up to $100 \%$.

\section{Table 1: Frequency of error types $\mathrm{N}=79$}

\section{Error type Frequency/percentage}

Dose at wrong time. . . . . . . . . . . . . . . . . . . . 25 (31.6\%) Missed dose. . . . . . . . . . . . . fast. . . . . . . . . . . . . . . . 3 (3.8\%) Wrong rate - too slow. . . $\ldots \ldots \ldots \ldots \ldots \ldots \ldots \ldots \ldots \ldots \ldots \ldots$ $\ldots \ldots \ldots \ldots \ldots 3(3.8 \%)$ Overdosage. . . . . . . . . . . . . . . . $\ldots \ldots 2$ ( $2.5 \%)$ Underdosage. . . . . . . . . . . . . . . . . . . . . . . . . ( 2.5\%) Extra dose. . . . . . . . . . . . . . . . . 1 1.3\%) Wrong strength. $\ldots \ldots \ldots \ldots \ldots \ldots \ldots \ldots \ldots \ldots \ldots \ldots \ldots \ldots \ldots)$

The most common errors were administering a dose of medicine at the wrong time and missing a dose of medication.

Table 2: Frequency of causes contributing to error

\section{Written cause Frequency/percentage}

Illegible handwriting. . . . . . . . . . . . . . . . . . . . . 13 (16.5\%) Misread

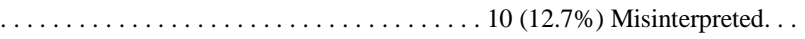
$\ldots \ldots \ldots \ldots \ldots \ldots \ldots \ldots \ldots \ldots$ (8.9\%) Misplaced decimal . . . . . . . $\ldots \ldots \ldots \ldots \ldots \ldots 6$ ( 7.6\%) Misunderstood abbreviations. . . . . . . $\ldots \ldots \ldots \ldots 5$ ( 6.3\%) Proprietary name confusion. . . . . . . . . . . .... 4 4 (5.1\%) Similar name confusion. . . . . . . . . . . . . . . . 4 4 ( $5.1 \%$ ) Incorrect patient ID on order $\ldots \ldots \ldots \ldots \ldots \ldots \ldots$ (3.8\%) Similar packaging confusion. . . . . . . . . . . . . . . . 2 ( 2.5\%) Name confusion. ................... (1.3\%)

The frequency and type of written mis-communication reported by nurses as causes of medication errors is presented in
Table 2. Illegible handwriting and misreading were the most common causes.

The frequency and type of human factors reported by nurses as causes of medication errors are presented in Table 3. Stress and fatigue were reported as the most common human causes of errors.

\section{Table 3: Frequency of human factors as causes}

\section{Human cause Frequency/percentage}

Stress/high workload. . . . . . . . . . . . . . . . . . 20 (25.3\%)

Fatigue/lack of sleep . . . . . . . . . . . . . . . . . . . 13 (16.5\%)

Knowledge deficit. . . . . . . . . . . . . . 7 ( $8.9 \%)$ Dose

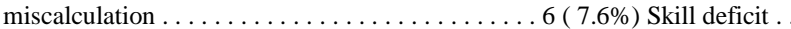

$\ldots \ldots \ldots \ldots \ldots \ldots$ (6.3\%) Confronting /

intimidating behaviour. . . . . . . . $3.8 \%$ (3.8)

The frequency and type of environmental factors reported by nurses as causes of medication errors are presented in Table 4. Interruptions and distractions were the major environmental issues.

\section{Table 4: Frequency of environmental factors as causes}

\section{Contributing cause Frequency/percentage}

Interruptions and distractions . . . . . . . . . . . . 20 (25.3\%) Poor communication between nurses/doctors . . . . . . . 10 (12.7\%) Staffing lack of other nurse. . . . . . . . . . . . 8 (10.1\%) Working with inexperienced nurse. . . . . . . . . . . 8 (10.1\%) Lighting. . . . . . .

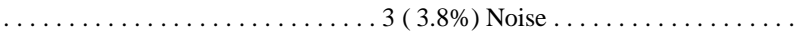

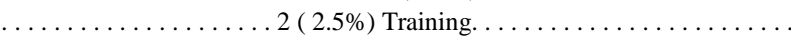
$\ldots \ldots \ldots \ldots 2(2.5 \%)$

The reporting behaviour of nurses who were involved in medication errors is presented in Table 5 . The feedback received following the reporting of the medication error was reported to be helpful, suggesting that the error was unlikely to be inadvertently repeated.

\section{Table 5: Frequency of reporting behaviours}

\section{Reporting behaviours Frequency/percentage}

Medication error reported . . . . . . . . . . . 10 (12.7\%) Verbal

feedback received. . . . . . . . . . . . . . . 10 (12.7\%) Helpful

feedback . . . . . . . . . . . . . . . . . . . . . . . 10 (12.7\%) Written

feedback received.

$.4(5.1 \%)$

The impact that the administration of medications had on the professional practice of registered nurses is presented in Table 6 (see page 32). Twenty nine percent of respondents either agreed or strongly agreed that they needed further training in medication administration. This was partially off-set by $96 \%$ of respondents indicating they had confidence when administering medications. Fifty seven percent of respondents indicated they have time to spend with patients when administering medications. Results showed that $89 \%$ of nurses felt comfortable reporting a medication error. 


\section{Table 6: Responses to questions about professional issues}

\section{Professional issues SA A U D SD}

I am comfortable reporting 3337251 a medication error (42\%) (47\%) (3\%) (6\%) $(1 \%)$

I have confidence when 3046210 administering medications (38\%) (58\%) (3\%) (1\%)

I refer to information when 2847120 administering medications (35\%) (60\%) (1\%) (3\%)

I have autonomy when 1943852 administering medications (24\%) (54\%) (10\%) (6\%) $(3 \%)$

I refer to a colleague when 1852260 administering medications (23\%) (66\%) (3\%) $(8 \%)$

I have satisfaction when 14471321 administering medications (18\%) (59\%) (17\%) (3\%) (1\%)

I have time to spend with patients 113415115 when administering medications (14\%) (43\%) (19\%) (14\%) (6\%)

I need further training in 51912356 medication administration (5\%) (24\%) (15\%) (44\%) (8\%)

I feel stressed when 1554918 administering medications (1\%) (6\%) (6\%) (62\%) (23\%)

SA = Strongly agree; $A=$ Agree $; U=$ Unsure $; D=$ Disagree; $S D=$ Strongly disagree

\section{Limitations}

The sample for this study was small and drawn from six clinical areas of a regional hospital, thus the findings may not be generalisable to registered nurses in other clinical settings. The instrument developed to conduct the survey may not have included all possible causes of medication errors; there could be additional causes.

\section{Discussion}

Four of the seven medication errors identified by Benner et al (2002) were reported in this study. The most frequently reported error was administration of medications at the wrong time. This finding concurs with Walker and Lowe (1998) who found that nurses did not consider reporting medication given one hour late as an error and were more likely to report a medication error when they believed patient safety may have been compromised. Perhaps the frequency of this occurrence has lulled nurses into accepting it as 'normal' practice. Whatever the reason, there is a need for all nurses in an organisation to be able to identify what constitutes a medication error, and when and to whom it should be reported.

A possible explanation for delays in the administration of medications may be found in the frequency that respondents reported illegible handwriting as a cause for making errors. One may assume that even if the illegible handwriting does not result in a mistake, there would be a substantial period of time devoted, and potentially stress experienced, in clarifying unclear written orders.

Clearly, these errors are linked to documentation issues and have the potential to cause a patient to miss a dose of medication or a treatment and can confuse, misrepresent, or mask a patient's true condition (Fox-Young 2003). It may be stating the obvious that electronic prescribing would substantially reduce the incidence of medication errors and risk to patients caused by illegible handwriting and related causes.

A related issue was the relatively high incidence of reported incidents about confusing the name of the medication, proprietary name confusion, similar packaging and similar name confusion. Clearly, this may have some implications for the manufacturers of drug products; assistance from the pharmacy in marking packages that have similarities to others could be explored. It may also be useful to provide physicians, nurses and pharmacists with orientation and periodic education on ordering, dispensing, administering and monitoring medications.

Although somewhat speculative, the causes for some medication errors lie in the area of knowledge deficit identified by respondents to the questionnaire. The Nurses Board of Victoria (NBV) expects that nurses only perform those clinical activities for which they have been educationally prepared and have demonstrated competence. Likewise, Naphine (in Jarman et al 2002) argued that nurses are individually accountable for their own actions and have the responsibility to protect patients from harm arising from medication errors. Both the organisation and the individual nurse have a responsibility to ensure individual medication administration competence. Nurses in this study identified willingness and need for more training in the area of medication administration and recognised their own knowledge deficits.

Associated with knowledge deficits, are the human and environmental factors identified by the nurses as contributory causes of medication errors. These include the high incidence of reported stressful workloads, fatigue and lack of sleep. This result is consistent with those reported by Osborne et al (1999) who reported these factors as the second most common cause of errors, and with Mayo and Duncan (2004) who reported them as the third highest ranked cause. In the current study, interruptions and distractions were ranked highest. This finding is in contrast to Osborne et al who ranked it fourth and Mayo and Duncan who ranked it second, but supports the findings of Gladstone (1995) who also ranked it as the major cause of error. Clearly, there is a need to support nurses in performing administration of medications. This would require an identification of strategies that could reduce the human and environmental impact on staff.

Ten respondents formally reported a medication error and all 10 indicated that they had received helpful verbal feedback. This finding is in contrast to Walker and Lowe's (1998) study, showing that nurses frequently said self-preservation was a major reason for nurses choosing not to formally report medication errors. The result in the current study emphasises the continuing need for health organisations to have a no-blame response at all levels of the organization and further supports the need for organisations to focus on a systems approach for reducing errors, rather than a person-centred 
Medication errors and professional practice of registered nurses approach (Anderson \& Webster 2001). Walker and Lowe (1998) also pointed out that attention to individuals rather than processes can result in nurses having a genuine fear of getting into trouble and receiving a reprimand from those in authority. There is little doubt that non-punitive incident self-reporting, which includes reports of near misses in addition to actual incidents, decreases the risk of serious consequences for the patient and identifies contributing factors that may be addressed by the organisation to prevent reoccurrences.

\section{Professional issues}

In addition to identifying medication errors and their causes, this study set out to describe professional issues associated with nurses administering medications. Results obtained from this section of the questionnaire support nurses' responses to types and causes of errors and also written comments. For example, respondents recognised that they needed further training in medication administration, which supports Shea's (1999) recommendation for nurse educators to provide in-service education relating to medication. This is an important professional issue for not only individual nurses, but has implications for the organisation in providing continuing education and assessing competence of their nurses to administer medicines.

Although percentages are relatively high for other professional issues such as autonomy and satisfaction, they are both below the $80 \%$ level, indicating room for improvement in these areas.

An important professional issue for nurses is that only a slight majority indicated they have time to spend with patients when administering medications, which may give the impression that medication rounds are somewhat rushed.

It is reassuring to report that nurses refer to other colleagues and information when administering medications and they would feel comfortable reporting a medication error. These results point to an organisational culture that encourages nurses to report and professional standards of accountability for nurses who recognise the importance of putting patient safety first.

\section{Conclusions}

The administration of medications is one of the most frequently occurring and highest-risk procedures in nursing. The organisation and those nurses employed within it share an accountability to ensure safe administration of medications to patients. Nurses should be aware of the potential for, and occurrence of, medication errors in settings where human and environmental factors interact. Added to this is the inherent degree of stress associated with nursing and the competing demands upon nurses' time and attention. If nurses are also experiencing tiredness or fatigue, the possibility for making mistakes is exacerbated. These factors contribute to a potent mix wherein errors, even for the most diligent nurse, are almost to be expected. It is imperative that nurses base their clinical practice on Clinical Practice Guidelines that set the minimum requirements for medication administration. It is also reasonable that the organisation put in place strategies that remove or reduce contributing factors to causes of medication errors. Medication errors are not the sole responsibility of any single professional group, therefore, collaboration with other health professionals is central to putting in place processes, policies, strategies and systems that will reduce their occurrence. Shea (1999) concluded that the multidisciplinary nature of medication errors called for a multidisciplinary approach in reducing errors. As medication errors will occur in the future there is a need for all health professionals to continually monitor the systems that have been put in place. There is a need in providing in-service and continuing education programs for nurses in medication administration. There is also a need to inform medical practitioners of the impact of illegible writing in relation to its perceived contribution to causes of medication errors.

\section{References}

Anderson JA, Webster CS 2001 A systems approach to the reduction of medication error on the hospital ward. Journal of Advanced Nursing 35(1):3441

Australian Council for Safety and Quality in Health Care 2001 Improving medication safety: report of a medication safety workshop. July, Government Publishers, Canberra

Benner P, Sheets V, Malloch K, Jamison D 2002 Individual, practice and system causes of errors in nursing: a taxonomy. Journal of Nursing Administration 32(10):509-523

Brown MM 2001 Managing medication errors by design. Critical Care Nursing Quarterly 24(3):77-97 Carothers NB 1998 Medication errors: the problem and its scope. International Journal of Trauma Nursing 4(3):104-108 Fox-Young S 2003 Nurse regulation and medications. Nursing Review Sept 6 Gladstone J 1995 Drug administration errors: a study into the factors underlying the occurrence and reporting of drug errors in a district general hospital. Journal of Advanced Nursing 22(4):628-637

Jarman H, Jacobs E, Zielinski V 2002 Medication study supports registered nurses' competence for single checking. International Journal of Nursing Practice 8:330-335

Leape LL, Bates DW, Cullen DJ, Cooper J, Demonaco HJ, Gallivan T et al 1995 Systems analysis of adverse drug events. Journal of the American Medical Association 274:35-43

Mayo AM, Duncan D 2004 Nurse perceptions of medication errors: what we need to know for patient safety. Journal of Nursing Care Quality 19(3):209-217 NCCMERP 1998 About medication errors. Retrieved January 212004 from the World Wide Web: http://www.nccmerp.org Nurses Board of Victoria 1995 General statement for nurses undertaking clinical practice/procedure. Nurses Board of Victoria, Melbourne Osborne J, Blais K, Hayes JS 1999 Nurses' perceptions: when is it a medication error? Journal of Nursing Administration 29(4):33-38

Phillips J, Beam S, Brinker A, Holquist C, Honig P, Lee LY, Pamer C 2001 Retrospective analysis of mortalities associated with medication errors. American Journal Health-System Pharmacy 58:1835-1841

The Royal Melbourne Hospital Inquiry (RMH) 2002 Health Services Commissioner, Melbourne, Victoria Reason J 2000 Human error: models and management. British Medical Journal 320:768-770

Ross LM, Wallace J, Paton JY 2000 Medication errors in a paediatric teaching hospital in the UK: five years operational experience. Archives of Disease in Childhood 83:492-497

Shea E 1999 Factors contributing to medication errors: a literature review Journal of Clinical Nursing 8:496-504 Walker SB, Lowe MJ 1998 Nurses' views on reporting medication incidents. International Journal of Nursing Practice 4:97-102 